\title{
TREATMENT OF CLASS I GINGIVAL RECESSION DEFECTS USING SINGLE FLAP APPROACH AND PLATELET RICH FIBRIN- A CASE REPORT
}

\author{
Amera Ayubi Parveen'1, Shivaprasad Bilichodmath², Nalini M. S3, Paunami Paul4, Ume Sameera ${ }^{5}$
}

1 Postgraduate Student, Department of Periodontology, Rajarajeswari Dental College and Hospital, Bangalore, Karnataka.

2Professor, Department of Periodontology, Rajarajeswari Dental College and Hospital, Bangalore, Karnataka.

${ }^{3}$ Reader, Department of Periodontology, Rajarajeswari Dental College and Hospital, Bangalore, Karnataka.

${ }_{4}^{4}$ Postgraduate Student, Department of Periodontology, Rajarajeswari Dental College and Hospital, Bangalore, Karnataka.

${ }_{5}^{5}$ Postgraduate Student, Department of Periodontology, Rajarajeswari Dental College and Hospital, Bangalore, Karnataka.

HOW TO CITE THIS ARTICLE: Parveen AA, Bilichodmath S, Nalini MS, et al. Treatment of class I gingival recession defects using single flap approach and platelet rich fibrin- a case report. J. Evolution Med. Dent. Sci. 2018;7(15):1925-1928, DOI: $10.14260 /$ jemds/2018/433

\section{PRESENTATION OF CASE}

A male patient aged 42 years reported to the hospital with the chief complaint of receding gums in the upper left front tooth region. There was no relevant medical, dental and family history. No deleterious oral habits. On physical examination, he appeared to be healthy and all his vital signs were within normal limit. On clinical examination, no significant extra-oral findings were noted. During clinical examination, Miller's class-I gingival recession was noticed with maxillary left lateral incisor and left canine i.e. with \#22 and \#23. Gingival recession of $3 \mathrm{~mm}$ were recorded with teeth 22 and 23 respectively (Figure 1). Gingival biotype was thin and width of attached gingiva was adequate. Whole surgical procedure was explained to the patient and written consent was obtained. Complete blood investigations were done before surgical procedure and scaling, and root planing was carried out. Oral hygiene instructions were given mainly in terms of proper brushing technique. Three weeks following this initial therapy, the periodontal re-evaluation was done for oral hygiene maintenance and to examine gingival tissue response to the initial therapy. After reevaluation, surgical procedure was carried out. An effective plaque control, good initial healing and proper defect anatomy was achieved; hence, a regenerative therapy was the treatment modality of choice. To correct this recession defect, single flap approach combined with platelet rich fibrin (PRF) was carried out. The single flap approach (SFA) is a simplified surgical procedure for treatment of recession. ${ }^{1}$ The basic underlying principle of the SFA consists of the elevation of a limited mucoperiosteal flap to allow access to the defect from either the buccal or oral aspect only, depending on the main buccal/ oral extension of the lesion (as assessed by preoperative bone sounding), allowing the interproximal supracrestal gingival tissues to remain intact. Aims behind the use of PRF in this surgical procedure were to correct the thin gingival biotype in recession area and to improve wound healing.

'Financial or Other Competing Interest': None.

Submission 03-03-2018, Peer Review 28-03-2018,

Acceptance 02-04-2018, Published 09-04-2018.

Corresponding Author:

Amera Ayubi Parveen,

Postgraduate Student,

Rajarajeswari Dental College and Hospital,

Rajarajeswari Engineering Ladies Hostel,

\#14, Ramohalli Cross, Kumbalgodu, Mysore Road,

Bangalore-560074, Karnataka, India.

E-mail: dr.ameraayubi@gmail.com

DOI: $10.14260 /$ jemds $/ 2018 / 433$

\section{(c) (1) $(9)$}

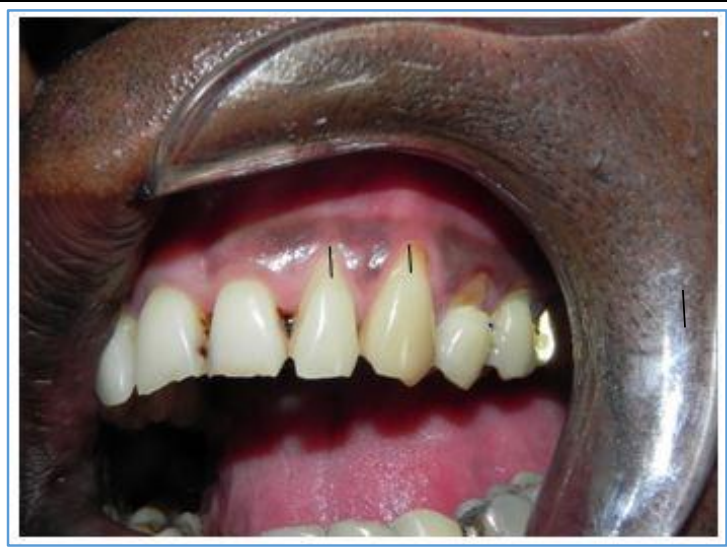

Figure 1. Gingival Recession of $3 \mathrm{~mm}$ with 22 and 23 respectively recorded at Baseline (Shown by Black Lines)

\section{MANAGEMENT/ PRF Protocol}

Before proceeding to surgical procedure, the PRF was prepared following the protocol developed by Choukroun et al. ${ }^{2} 10 \mathrm{~mL}$ of intravenous blood (by a venipuncture of the antecubital vein) was collected into two test tubes (each containing $5 \mathrm{~mL}$ of blood) without anticoagulant and immediately centrifuged at 3000 revolutions/ min for 10 minutes. At the end of centrifugation three layers were seen, the top layer containing supernatant serum, the fibrin clot at the middle layer and the bottom layer containing the red blood corpuscles (RBC). The fibrin clot was easily separated from the RBC base (preserving a small RBC layers) using sterile tweezers and scissors ${ }^{3}$ (Figure 2). It was placed in a sterile dappen dish and was left aside. Before use it was slightly squeezed with the gauze piece to remove its serum content and prepared in the form of membranes (Figure 3).

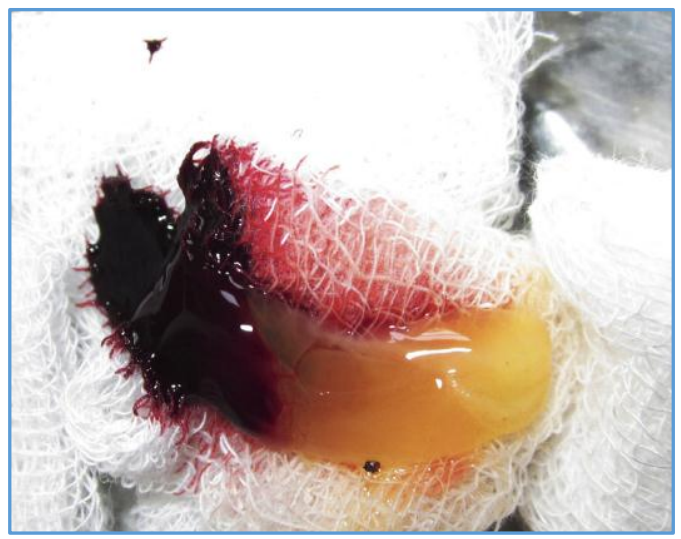

Figure 2. PRF Clot Removal 


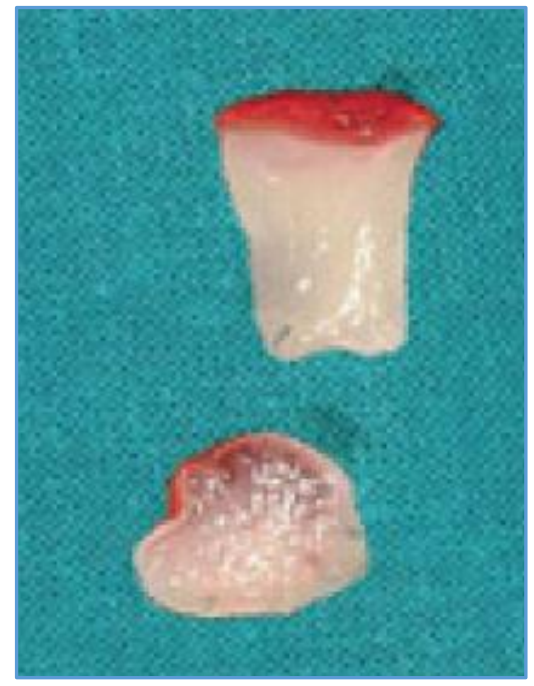

Figure 3. PRF Membrane obtained by Driving away the Serum from the Clot

\section{Surgical Procedure}

After giving local anaesthesia (1: 200000 adrenaline), a split thickness single flap approach was performed on the buccal aspect of the teeth being treated. Initially, an intrasulcular incision was given from mesial side of \#22 to the distal side of \#23. A butt-joint incision at the base of the papilla at the site of the defect was performed and two vertical incisions were given, i.e. from mesial line angle of \#22 and distal line angle of \#23 extending beyond the mucogingival junction. All incisions were made using blade \#15. A split thickness flap was raised carefully on the buccal aspect only. Freely movable flap was advanced coronally with its margin located on enamel and the vertical sutures were used to create an envelope, which was interposed with the previously prepared PRF membranes and was positioned and stabilised at the desired position. Suturing was done using resorbable sutures (Figure 4-7). Gentle pressure was applied at the surgical site with moistened gauze to achieve haemostasis and followed by periodontal dressing. Patients were prescribed antibiotics and analgesics (Cap. Amoxicillin 500 mg, TDS and Tab. Paracetamol + Ibuprofen, TDS for 5 days). Post-operative instructions were given and patient was informed to report after 10 days for suture removal (Figure 8). Professional scaling and oral hygiene reinforcement were provided at each follow-up visit whenever indicated. Patient was followed up for 9 months and $100 \%$ of root coverage was observed (Figure 9).

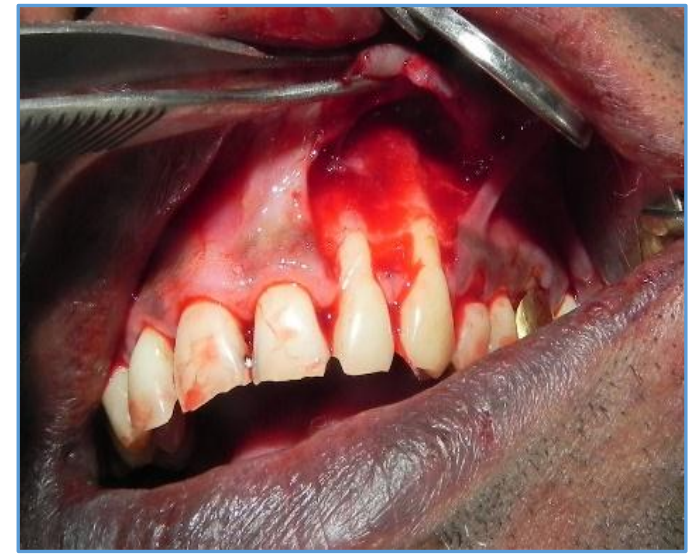

Figure 4. Split Thickness SFA Performed

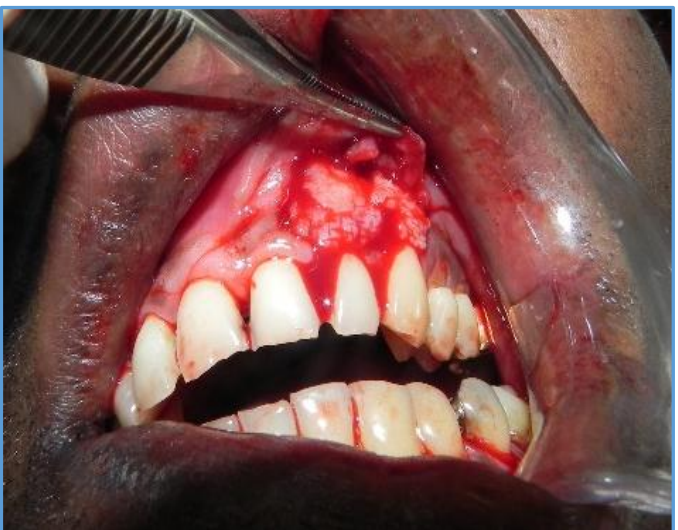

Figure 5. PRF Membrane placed in the Recession Defects

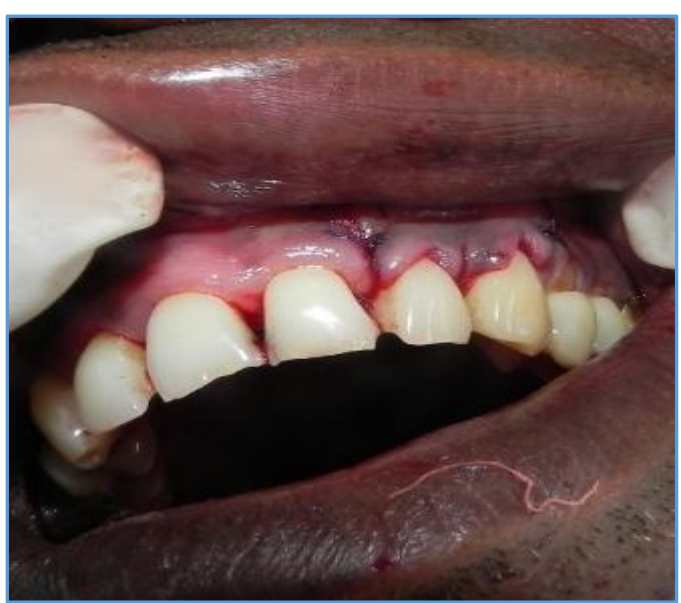

Figure 6. Sutures Placed

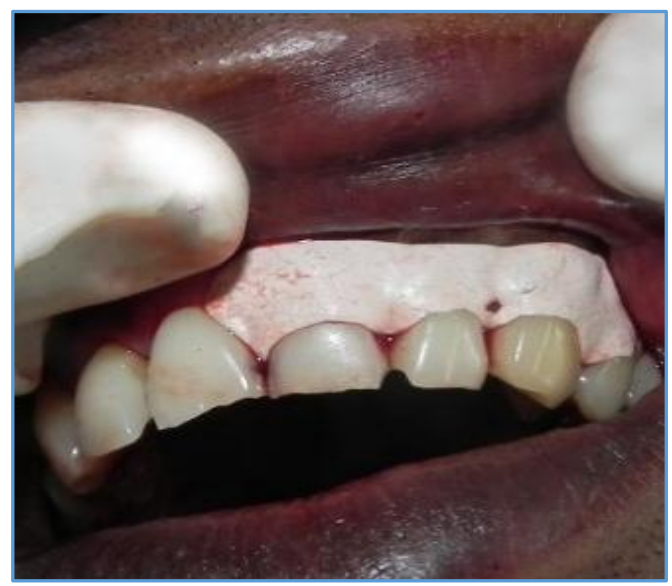

Figure 7. Periodontal Pack Placed

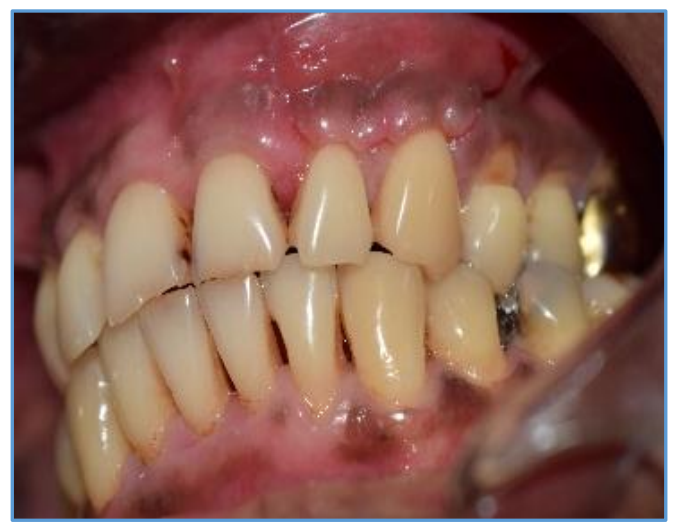

Figure 8. 10 Days Post-Operative 


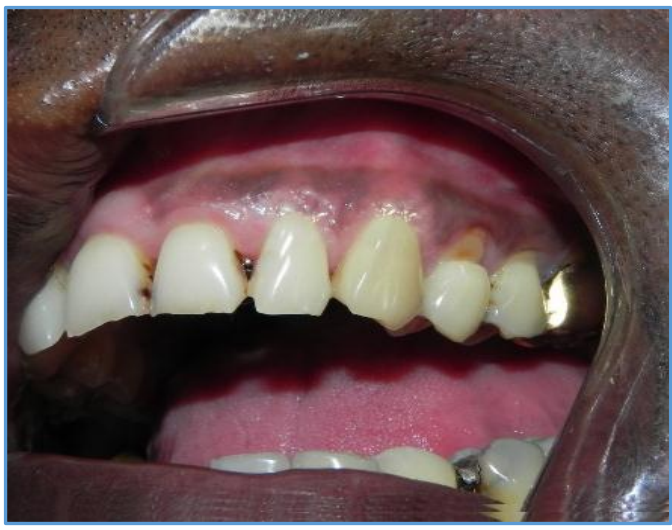

Figure 9. 9 Months Post-Operative

\section{DISCUSSION}

Treatment of gingival recession is becoming an important issue in clinical periodontology due to the increasing demand for cosmetic treatment. Problems related particularly to the fact that very often the most coronal millimetres of the recession are observed while smiling. Thus, only surgical procedures that provide the clinician with a very high percent of complete root coverage should be included in the mucogingival plastic surgical techniques. Recession defect as shown in this case report could be treated with pedicle soft tissue grafts, free soft tissue grafts or combination of the two. Among the pedicle grafts, the SFA which is one of the modifications of CAF is a valid surgical option to cover exposed root surfaces. It has many advantages over other surgical procedures used to treat gingival recessions. It does not require a separate surgical site to obtain a graft. The tissue of the pedicle provides a perfect colour and contour match with the surrounding tissue. The procedure is simple to perform and does not require an extended surgical or recovery time. ${ }^{4}$ Therefore, in the presented case report, PRF was used along with SFA. PRF also promotes more rapid attachment to the tooth with stable result. In addition, PRF slows down the blood activation process which could induce an increased leukocyte degranulation and cytokine release from pro-inflammatory mediators such as interleukin (IL)-1 $\beta$, IL- 6 and tumour necrosis factor- $\alpha$ to anti-inflammatory cytokines such as IL-4, different growth factors like transforming growth factor- $1 \beta$, platelet derived growth factor- $\alpha \beta$, and vascular endothelial growth factor and glycoproteins (thrombospondin-1) over more than 7 days. Leukocytes seem to have a strong influence on growth factor release, immune regulation, anti-infectious activities and matrix remodelling during healing. Moreover, the fibrin matrix itself shows mechanical adhesive properties and biologic functions like fibrin glues. It maintains the flap in a high and stable position, enhances neoangiogenesis, reduces necrosis and shrinkage of the flap and guarantees maximal root coverage. ${ }^{5}$ Aroca et al presented mean root coverage of 80.7\% after 6 months, utilising PRF. 6 Thamaraiselvan $M$ et al compared CAF with and without PRF in the treatment of isolated Miller's class-I and class-II gingival recession. The CAF group showed a non-significant gingival thickness (GTH) increase of $0.03 \pm 0.04 \mathrm{~mm}$, which is similar to other studies. Interestingly, the addition of PRF to CAF resulted in a $0.30 \pm 0.10 \mathrm{~mm}$ GTH increase, which was statistically significant when compared both within and between the groups. $^{3}$ Baiju RM et al used PRF along with CAF to treat gingival recession of around 2 - $3 \mathrm{~mm}$ with \#22 and \#23 and superior results were obtained after the treatment with residual recession of only $1 \mathrm{~mm}$ remained with 23 after 6 months. ${ }^{5} \mathrm{~A}$ study by Corso et al have confirmed the successful use of PRF membranes in the management of both single and multiple gingival recession defects. ${ }^{7}$ In a similar study by Eren and Atilla accepted that the PRF method is practical and simple to perform. ${ }^{8}$ Additionally, they found PRF to be superior to subepithelial connective tissue graft, since it eliminates the requirement of a donor site. Baseline recession depth is important in determining the treatment outcome. Greater baseline gingival recession depth is always associated with decreased results in terms of root coverage. ${ }^{9}$ Gingival recession depth is not only a factor which determines the clinical outcome, other factors like root prominence, tooth position, vestibular depth, high frenal pull, gingival thickness as well as tooth brushing technique of the patient have to be considered and should be corrected before carrying out root coverage procedure. This enhances the treatment outcome and helps to maintain long-term results. Positioning the PRF at the CEJ may also favour initial root exposure. This was reported in $53 \%$ of single recessions treated with a bilaminar surgical technique. ${ }^{9}$ Thus, in the presented case report, addition of PRF to SFA helped to obtain favourable clinical outcome in terms of root coverage. Therefore, the effect of PRF on the establishment of a connective tissue attachment remains to be determined. Further evaluation of PRF to SFA is necessary to find out the type of healing, histologically as well as long-term follow-up of the clinical case.

\section{REFERENCES}

[1] Trombelli L, Simonelli A, Minenna L, et al. Simplified procedures to treat periodontal intraosseous defects in esthetic areas. Periodontol 2000. 2018.

[2] Choukroun J, Adda F, Schoeffler C, et al. An opportunity in perio-implantology: the PRF (in French). Implantodontie 2001;42:55-62.

[3] Thamaraiselvan M, Elavarasu S, Thangakumaran S, et al. Comparative clinical evaluation of coronally advanced flap with or without platelet rich fibrin membrane in the treatment of isolated gingival recession. J Indian Soc Periodontol 2015;19(1):66-71.

[4] Ozturan S. Treatment of gingival recession with coronally advanced flap procedure case report. IJBCS 2013;2:153-9.

[5] Baiju RM, Ahuja R, Ambili G, et al. Autologous plateletrich fibrin: a boon to periodontal regeneration-report of two different clinical applications. Health Sciences 2013;2:1-13.

[6] Aroca S, Keglevich T, Barbieri B, et al. Clinical evaluation of a modified coronally advanced flap alone or in combination with a platelet-rich fibrin membrane for the treatment of adjacent multiple gingival recessions: a 6 month study. J Periodontol 2009;80(2):244-52.

[7] Corso MD, Sammartino G, Ehrenfest DM. Clinical evaluation of a modified coronally advanced flap alone or in combination with a platelet-rich fibrin membrane for the treatment of adjacent multiple gingival recessions: a 6-month study. J Periodontol 2009;80(11):1694-7. 


\section{Jemds.com}

[8] Eren G, Atilla G. Platelet rich fibrin in the treatment of localized gingival recessions: a split mouth randomized clinical trial. Clin Oral Investig 2014;18(8):1941-8.

\section{Case Report}

[9] Zucchelli G, Amore C, Sforza NM, et al. Bilaminar techniques for the treatment of recession-type defects. A comparative clinical study. J Clin Periodontol 2003;30(10):862-70. 